\title{
Implications of an Improved Neutron-Antineutron Oscillation Search for Baryogenesis: A Minimal Effective Theory Analysis
}

\author{
Christophe Grojean, ${ }^{1,2}$ Bibhushan Shakya, ${ }^{3,4}$ James D. Wells, ${ }^{4}$ and Zhengkang Zhang ${ }^{4}$ \\ ${ }^{1}$ Deutsches Elektronen-Synchrotron (DESY), 22607 Hamburg, Germany \\ ${ }^{2}$ Institut für Physik, Humboldt-Universität zu Berlin, 12489 Berlin, Germany \\ ${ }^{3}$ Department of Physics, University of Cincinnati, Cincinnati, Ohio 45221, USA \\ ${ }^{4}$ Leinweber Center for Theoretical Physics (LCTP), University of Michigan, Ann Arbor, Michigan 48109, USA
}

(Received 19 July 2018; revised manuscript received 26 September 2018; published 23 October 2018)

Future neutron-antineutron $(n-\bar{n})$ oscillation experiments, such as at the European Spallation Source and the Deep Underground Neutrino Experiment, aim to find first evidence of baryon number violation. We investigate implications of an improved $n-\bar{n}$ oscillation search for baryogenesis via interactions of $n-\bar{n}$ mediators, parametrized by an effective field theory (EFT). We find that even in a minimal EFT setup there is overlap between the parameter space probed by $n-\bar{n}$ oscillation and the region that can realize the observed baryon asymmetry of the Universe. The mass scales of exotic new particles are in the teraelectron-volt-peta-electron-volt regime, inaccessible at the LHC or its envisioned upgrades. Given the innumerable high energy theories that can match, or resemble, the minimal EFT that we discuss, future $n-\bar{n}$ oscillation experiments could probe many viable theories of baryogenesis beyond the reach of other experiments.

DOI: 10.1103/PhysRevLett.121.171801

Introduction.-The search for physics beyond the standard model (BSM) requires effort at both high energy and intensity frontiers. In this regard, a particularly powerful probe is offered by rare processes that violate (approximate) symmetries of the SM, such as baryon and lepton numbers $(B$ and $L)$, which can be inaccessible to high energy colliders but within reach of low-energy experiments. A well-known example is proton decay, whose nonobservation leads to strong constraints on $\Delta B=\Delta L=$ \pm 1 new physics even at the scale of grand unified theories (GUTs), $\sim 10^{16} \mathrm{GeV}[1,2]$.

Baryon and lepton number violation are intricately tied to one of the outstanding puzzles in fundamental physics, the origin of the baryon asymmetry in the Universe. If baryogenesis occurs at temperatures above the weak scale, $B-L$ violation is required to avoid washout by electroweak sphalerons. In this regard, constraints from proton decay (which conserves $B-L$ ) are not applicable. Here we consider instead $B$-violating, $L$-conserving new physics at an intermediate (sub-GUT) scale so that baryogenesis may proceed both above and below weak scale temperatures. From the low-energy point of view, effects of heavy new particles are encoded in higher dimensional operators

Published by the American Physical Society under the terms of the Creative Commons Attribution 4.0 International license. Further distribution of this work must maintain attribution to the author(s) and the published article's title, journal citation, and DOI. Funded by SCOAP ${ }^{3}$. in an effective field theory (EFT), where $B$-violating, $L$-conserving interactions can appear first at the dimension-9 level [3], in the form of $|\Delta B|=2, \Delta L=0$ operators. In this case, neutron-antineutron $(n-\bar{n})$ oscillation (see Ref. [4] for a recent review) is well placed to search for $B$-violating phenomena and shed light on baryogenesis [5].

Current measurements constrain the free neutron oscillation time to be $\tau_{n \bar{n}} \gtrsim 10^{8} \mathrm{~s}[6,7]$. Upcoming experiments, in particular at the European Spallation Source (ESS) and also potentially the Deep Underground Neutrino Experiment (DUNE), are poised to improve the reach up to $10^{9-10} \mathrm{~s}$ [8-12]. As we will see in detail below, such numbers translate into new physics scales of roughly $\left(\tau_{n \bar{n}} \Lambda_{\mathrm{QCD}}^{6}\right)^{1 / 5} \sim O\left(10^{5-6} \mathrm{GeV}\right)$, well above the energies directly accessible at existing or proposed colliders. Discussions of the physics implications of a potential $n-\bar{n}$ oscillation discovery, in particular for baryogenesis, are therefore both important and timely.

The purpose of this Letter is to explore the connection between $n-\bar{n}$ oscillation and baryogenesis in the context of a minimal EFT extension of the SM that realizes direct low scale baryogenesis from $B$-violating decays of new particles mediating $n-\bar{n}$ oscillation. While there exist numerous baryogenesis frameworks, such as electroweak baryogenesis [13], Affleck-Dine baryogenesis [14], and leptogenesis [15] (see, e.g., [16-24] for reviews), the choice of our minimal EFT is motivated from the bottom up by imminent improvements in $n-\bar{n}$ oscillation searches. Despite being simplistic, this minimal setup provides a useful template to identify viable baryogenesis scenarios-which may be 
realized in a similar manner in more complex and realistic theories-that are compatible with an $n-\bar{n}$ oscillation signal within experimental reach (for discussions of some other baryogenesis scenarios that can also involve $n-\bar{n}$ oscillation signals, see, e.g., [25-43]).

Improved $n-\bar{n}$ oscillation searches and sensitivity to the scale of new physics. - Neutron-antineutron oscillation has been searched for in the past with both free neutrons $[6,44,45]$ and neutrons bound inside nuclei [7,46-50]. Among free neutron oscillation searches, the Institut Laue-Langevin experiment [6] sets the best limit to date on the oscillation time, $\tau_{n \bar{n}}>0.86 \times 10^{8} \mathrm{~s}$ at $90 \%$ C.L. Among intranuclear searches, Super-Kamiokande (Super-K) [7] provides the best limit, which, after correcting for nuclear effects, corresponds to $\tau_{n \bar{n}}>2.7 \times 10^{8} \mathrm{~s}$ at $90 \%$ C.L. for the free neutron oscillation time. Improved $n-\bar{n}$ oscillation searches with both free and bound neutrons are under consideration, with sensitivities up to $10^{9-10} \mathrm{~s}$ envisioned at the ESS and DUNE [8-12].

We now elucidate the connection between $\tau_{n \bar{n}}$ and the new physics scale in the EFT context. The lowest dimension effective operators contributing to $n-\bar{n}$ oscillation at tree level are dimension-9 operators of the form $O_{n \bar{n}} \sim$ $(u u d d d d)$. The classification of these operators dates back to the 1980s [51-55] and was refined recently in [56], which established an alternative basis that is more convenient for renormalization group (RG) running. A concise review of the full set of tree-level $n-\bar{n}$ oscillation operators is given in the Supplemental Material [57]. In what follows, we focus on one of these operators for illustration,

$$
\begin{aligned}
\mathcal{L} \supset & c_{1} \frac{1}{2} \epsilon_{i j k} \epsilon_{i^{\prime} j^{\prime} k^{\prime}}\left(\bar{u}_{i}^{c} P_{R} d_{j}\right)\left(\bar{u}_{i^{\prime}}^{c} P_{R} d_{j^{\prime}}\right)\left(\bar{d}_{k}^{c} P_{R} d_{k^{\prime}}\right) \\
& + \text { H.c. }, \\
\text { with } \quad c_{1} \equiv & \left(\Lambda_{n \bar{n}}^{(1)}\right)^{-5} .
\end{aligned}
$$

Here $u, d$ are SM up and down quark fields, respectively, and $u^{c}, d^{c}$ are their charge conjugates. $i^{(\prime)}, j^{(\prime)}, k^{(\prime)}$ are color indices, and "H.c." denotes Hermitian conjugate. The operator suppression scale $\Lambda_{n \bar{n}}^{(1)}$ is generally a weighted (geometric) average of new particle masses, modulo appropriate powers of couplings and loop factors.

If the operator is generated by integrating out new particles at a high scale $M$, computing $\tau_{n \bar{n}}$ requires RG evolving the EFT down to a low scale $\mu_{0}$ (usually chosen to be $2 \mathrm{GeV}$ ), where it can be matched onto lattice QCD. The leading contribution to $\mathrm{RG}$ rescaling reads $[55,56]$

$$
\begin{aligned}
\frac{c_{1}\left(\mu_{0}\right)}{c_{1}(M)} & =\left(\frac{\alpha_{s}^{(4)}\left(m_{b}\right)}{\alpha_{s}^{(4)}\left(\mu_{0}\right)}\right)^{6 / 25}\left(\frac{\alpha_{s}^{(5)}\left(m_{t}\right)}{\alpha_{s}^{(5)}\left(m_{b}\right)}\right)^{6 / 23}\left(\frac{\alpha_{s}^{(6)}(M)}{\alpha_{s}^{(6)}\left(m_{t}\right)}\right)^{2 / 7} \\
& =\{0.726,0.684,0.651,0.624\} \\
\text { for } M & =\left\{10^{3}, 10^{4}, 10^{5}, 10^{6}\right\} \mathrm{GeV} .
\end{aligned}
$$

Here $\alpha_{s}^{\left(n_{f}\right)}$ is the effective strong coupling with $n_{f}$ light quark flavors, whose value is obtained with the RUNDEC package [58]. Corrections from two-loop running as well as one-loop matching onto lattice QCD operators were recently computed [56] and are small, and they will be neglected in our calculations. No additional operators relevant for $n-\bar{n}$ oscillation are generated from RG evolution.

The $n \rightarrow \bar{n}$ transition rate is determined by the matrix element of the low-energy effective Hamiltonian between the neutron and antineutron states. Thus, once $\left\langle\bar{n}\left|O_{n \bar{n}}\left(\mu_{0}\right)\right| n\right\rangle$ are known, we can relate $\tau_{n \bar{n}}=\left|\left\langle\bar{n}\left|\mathcal{H}_{\text {eff }}\right| n\right\rangle\right|^{-1}$ to the six-quark operator coefficients. Recent progress in lattice calculations [59-61] has greatly improved the accuracy on $\left\langle\bar{n}\left|O_{n \bar{n}}\left(\mu_{0}\right)\right| n\right\rangle$ compared to previous bag model calculations $[53,54]$ often used in the literature. Using the central values presented in [61], and assuming that the operator in Eq. (1) gives the dominant contribution to $n-\bar{n}$ oscillation, we can translate the Super-K limit into $\Lambda_{n \bar{n}}^{(1)} \gtrsim 3.8 \times 10^{5} \mathrm{GeV}$ (for a representative RG rescaling factor of 0.7). An improvement on $\tau_{n \bar{n}}$ up to $10^{9}\left(10^{10}, 10^{11}\right) \mathrm{s}$ will correspond to probing $\Lambda_{n \bar{n}}^{(1)} \sim$ $4.9(7.8,12.4) \times 10^{5} \mathrm{GeV}$. These numbers are representative of the whole set of $n-\bar{n}$ oscillation operators and do not vary significantly with the starting point of RG evolution $M$ (see the Supplemental Material [57] for details).

A minimal EFT for $n-\bar{n}$ oscillation and baryogenesis.One of the simplest possibilities for generating the operator in Eq. (1) at tree level is with a Majorana fermion $X$ of mass $M$ that couples to the SM via a dimension- 6 operator of the form $\left(1 / \Lambda^{2}\right) X u d d$, which originates at an even higher scale $\Lambda \gg M$ via some UV completion that we remain agnostic about. A familiar scenario that realizes this EFT setup is supersymmetry (SUSY) with $R$-parity violation (RPV), where the bino plays the role of $X$ and the dimension-6 operator is obtained by integrating out squarks at a heavier scale. However, this simple EFT with a single BSM state does not allow for sufficient baryogenesis due to unitarity relations: in the absence of $B$-conserving decay channels, $X$ decay cannot generate a baryon asymmetry at leading order in the $B$-violating coupling, a result known as the Nanopoulos-Weinberg theorem [62] (see [63] for a recent discussion); meanwhile, $2 \rightarrow 2$ processes $u X \rightarrow \bar{d} \bar{d}$ and $\bar{u} X \rightarrow d d$ are forced to have the same rate and thus do not violate $C P$.

A minimal extension that can accommodate both $n-\bar{n}$ oscillation and the observed baryon asymmetry involves two Majorana fermions $X_{1}, X_{2}$ (with $M_{X_{1}}<M_{X_{2}}$ ), each having a $B$-violating interaction $\left(1 / \Lambda^{2}\right) X u d d$. In addition, a $B$-conserving coupling between the two is necessary to evade constraints from unitarity relations. In the context of RPV SUSY, this corresponds to the presence of a wino or gluino in addition to the bino, which is known to allow for sufficient baryogenesis [63-65].

Guided by minimality, we assume that $X_{1,2}$ are both SM singlets and consider just one of the many possible 
$B$-conserving operators in addition to the two $B$-violating ones. Our minimal EFT thus consists of the following dimension- 6 operators that couple $X_{1,2}$ to the SM [66]:

$$
\begin{aligned}
\mathcal{L} \supset & \eta_{X_{1}} \epsilon^{i j k}\left(\bar{u}_{i}^{c} P_{R} d_{j}\right)\left(\bar{d}_{k}^{c} P_{R} X_{1}\right) \\
& +\eta_{X_{2}} \epsilon^{i j k}\left(\bar{u}_{i}^{c} P_{R} d_{j}\right)\left(\bar{d}_{k}^{c} P_{R} X_{2}\right) \\
& +\eta_{c}\left(\bar{u}^{i} P_{L} X_{1}\right)\left(\bar{X}_{2} P_{R} u_{i}\right)+\text { H.c., }
\end{aligned}
$$

with $\quad\left|\eta_{X_{1}}\right| \equiv \Lambda_{X_{1}}^{-2}, \quad\left|\eta_{X_{2}}\right| \equiv \Lambda_{X_{2}}^{-2}, \quad\left|\eta_{c}\right| \equiv \Lambda_{c}^{-2}$.

Both $X_{1}$ and $X_{2}$ mediate $n-\bar{n}$ oscillation-integrating them out at tree level gives

$$
c_{1}=\frac{1}{\left(\Lambda_{n \bar{n}}^{(1)}\right)^{5}}=\frac{1}{M_{X_{1}} \Lambda_{X_{1}}^{4}}+\frac{1}{M_{X_{2}} \Lambda_{X_{2}}^{4}} .
$$

This setup contains all of the necessary ingredients for baryogenesis [67]: the Lagrangian in Eq. (3) violates $B$ and $P$, while nonzero phases of $\eta_{X_{1}}, \eta_{X_{2}}$, and $\eta_{c}$ can lead to $C P$ violation; departure from equilibrium can occur in multiple ways, as we discuss below. Although a clear simplification, we expect the minimal set of operators in Eq. (3) to capture the generic qualitative features possible in a two $n-\bar{n}$ mediator setup, which can be realized in more complicated and realistic frameworks.

Calculation of the baryon asymmetry.-The relevant processes for baryogenesis include (1) $B$-violating processes: single annihilation $u X_{1,2} \rightarrow \bar{d} \bar{d}, d X_{1,2} \rightarrow \bar{u} \bar{d}$, decay $X_{1,2} \rightarrow u d d$, and off-resonance scattering $u d d \rightarrow \bar{u} \bar{d} \bar{d}$; and (2) $B$-conserving processes: scattering $u X_{1} \rightarrow u X_{2}$, coannihilation $X_{1} X_{2} \rightarrow \bar{u} u$, and decay $X_{2} \rightarrow X_{1} \bar{u} u$, as well as their inverse and $C P$ conjugate processes. $C P$ violation arises from interference between tree and one-loop diagrams in $u X_{1,2} \leftrightarrow \bar{d} \bar{d}, \quad u X_{1} \leftrightarrow u X_{2}$, and $X_{2} \leftrightarrow u u d$, and additionally from $u d d \leftrightarrow \bar{u} \bar{d} \bar{d}$ (in a way that is related to $X_{2} \leftrightarrow u u d$ by unitarity). In each case, $C P$ violation is proportional to $\operatorname{Im}\left(\eta_{X_{1}}^{*} \eta_{X_{2}} \eta_{c}\right) \sim \Lambda^{-6}$. We work at leading order in the EFT expansion, i.e., $O\left(\Lambda^{-4}\right)$ for the rates of $C P$ conserving processes and the $C P$-symmetric components of $C P$-violating processes, and $O\left(\Lambda^{-6}\right)$ for the $C P$ violating rates. We choose a mass ratio $M_{X_{2}} / M_{X_{1}}=4$, which maximizes $\Gamma\left(X_{2} \rightarrow u d d\right)-\Gamma\left(X_{2} \rightarrow \bar{u} \bar{d} \bar{d}\right)$ for fixed $M_{X_{2}}$.

We calculate the baryon asymmetry by numerically solving a set of coupled Boltzmann equations to track the abundances of $X_{1,2}$ and $B-L(B)$ above (below) $T=$ $140 \mathrm{GeV}$ (we assume that sphalerons are active when $T>140 \mathrm{GeV}$, resulting in $\left.Y_{B}=\frac{28}{79} Y_{B-L}\right)$. Our aim is to find regions of parameter space that can achieve the observed $Y_{B}=8.6 \times 10^{-11}[68,69]$, with suitable choice of $C P$ phases. Technical details of this calculation can be found in the Supplemental Material [57].
If all three operator coefficients have similar sizes, $\Lambda_{X_{1}} \sim \Lambda_{X_{2}} \sim \Lambda_{c}$, it is difficult to obtain the observed baryon asymmetry in the region of parameter space probed by $n-\bar{n}$ oscillation. For $M_{X_{1,2}} \gtrsim 10^{4} \mathrm{GeV}$, the $\Lambda$ 's that can be probed are sufficiently low for $X_{1,2}$ to remain close to equilibrium until their abundances become negligible, while efficient washout suppresses $B(-L)$ generation. For lower masses and higher $\Lambda$ 's, on the other hand, $X_{2}$ may freeze out with a significant abundance, and decay out of equilibrium at later times when washout has become inefficient, so that both limitations from the higher mass regime are overcome. However, its $C P$-violating branching fraction $\epsilon_{C P} \sim M_{X_{2}}^{2} / \Lambda^{2}$ is too small to generate the desired $Y_{B}$. We find that for $\Lambda_{X_{1}}=\Lambda_{X_{2}}=\Lambda_{c}$, the maximum $Y_{B}$ possible in the ESS-DUNE sensitivity region is $O\left(10^{-13}\right)$, well below the observed value.

Achieving the desired baryon asymmetry in the ESSDUNE reach region therefore requires hierarchical $\Lambda$ 's; such scenarios can arise if new particles in the UV theory that mediate the corresponding operators have hierarchical masses and/or couplings, or if the EFT operators are generated at different loop orders. We find compatible regions of parameter space in two distinct scenarios, one with late decays of $X_{2}$ and the other with earlier decays. These are schematically illustrated in Fig. 1 and discussed in turn below (a detailed analysis with benchmark numerical solutions is presented in the Supplemental Material [57]).

Late decay scenario. - For $\Lambda_{X_{2}} \sim \Lambda_{c} \gg \Lambda_{X_{1}}, n-\bar{n}$ oscillation is dominated by $X_{1}$ exchange and probes the $M_{X_{1}}-\Lambda_{X_{1}}$ parameter space (see Fig. 2). This hierarchy leads to weaker interactions for $X_{2}$ compared to the degenerate case, causing it to freeze out with a higher abundance $Y_{X_{2}}^{\text {fo }}$.

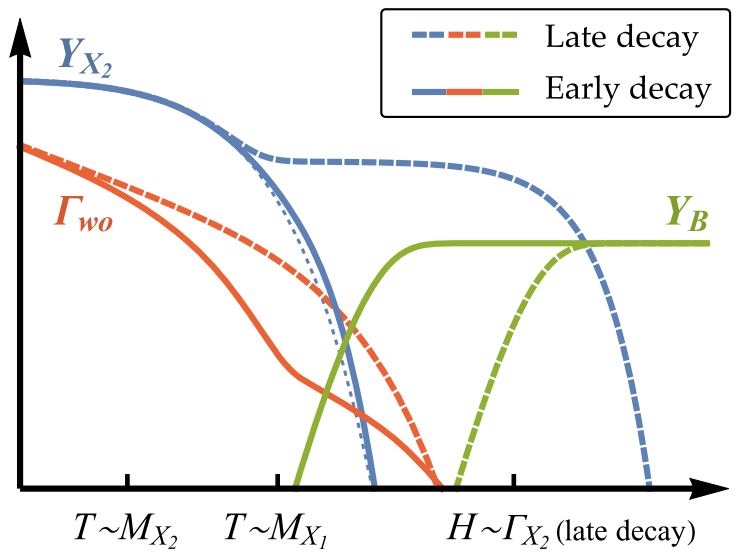

FIG. 1. Sketches of the evolution of the heavier $n-\bar{n}$ mediator abundance $Y_{X_{2}}$, washout rate $\Gamma_{\text {wo }}$, and baryon asymmetry $Y_{B}$ in the two scenarios considered in this Letter (arbitrary normalization). In the late decay scenario, the $n-\bar{n}$ mediator is long-lived and decays out of equilibrium to generate a baryon asymmetry. In the early decay scenario, departure from equilibrium (thin dotted curve) is small, but suppressed washout enables efficient baryogenesis. See the text for details. 


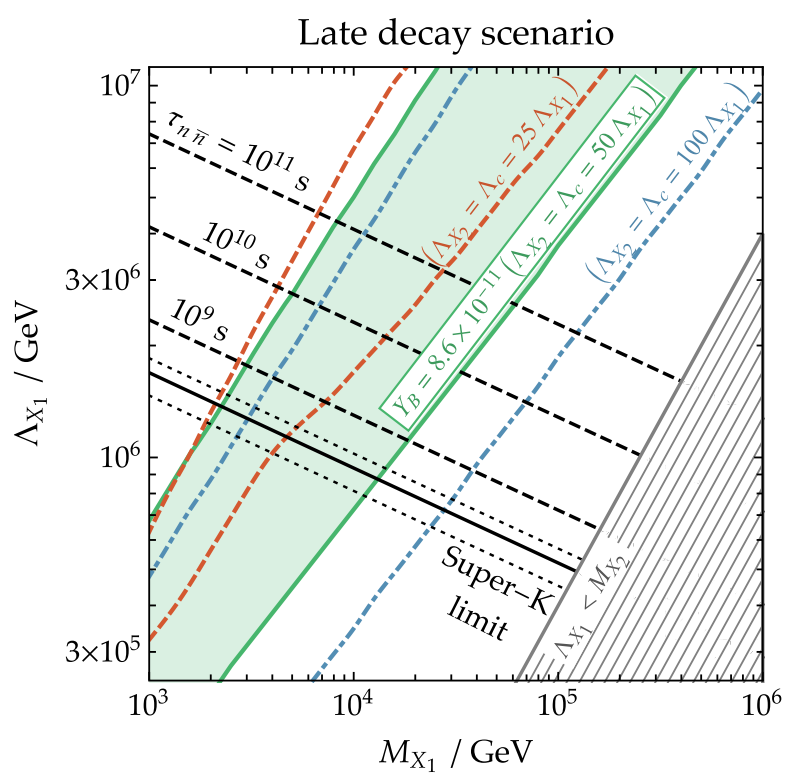

FIG. 2. Parameter space of the minimal EFT probed by $n-\bar{n}$ oscillation for the late decay scenario, assuming that $M_{X_{2}}=4 M_{X_{1}}$. The thicker (solid and dashed) black lines are obtained using central values of nuclear matrix elements from [61], while the thinner dotted lines show the effect of current lattice calculation uncertainty on the Super-K limit. For $\Lambda_{X_{2}}=\Lambda_{c}=50 \Lambda_{X_{1}}$, the green shaded region can accommodate $Y_{B}=8.6 \times 10^{-11}$. For $\Lambda_{X_{2}}=\Lambda_{c}=25 \Lambda_{X_{1}}\left(100 \Lambda_{X_{1}}\right)$, the viable region is between the dashed red (dotted-dashed blue) lines. The gray shaded region marks $\Lambda_{X_{1}}<M_{X_{2}}$, where EFT validity requires greater than $O(1)$ coupling.

Also, $X_{2}$ becomes long-lived and decays after washout processes have become ineffective, thereby creating substantial baryon asymmetry (see Fig. 1). In this case, its $C P$-violating branching fraction scales as $\epsilon_{C P} \sim$ $M_{X_{2}}^{2} \eta_{X_{1}} \eta_{X_{2}} \eta_{c} / \max \left(\eta_{X_{2}}^{2}, \eta_{c}^{2}\right) \sim M_{X_{2}}^{2} / \Lambda_{X_{1}}^{2}$ and does not decouple as $\Lambda_{X_{2}}$ and $\Lambda_{c}$ are both increased, enabling $Y_{B} \sim$ $Y_{X_{2}}^{\text {fo }} \epsilon_{C P}$ to reach the observed value.

Numerically, we find that this baryogenesis scenario is viable with $\Lambda_{X_{2}}, \Lambda_{c} \gtrsim 20 \Lambda_{X_{1}}$ in the parameter space probed by $n-\bar{n}$ oscillation. In Fig. 2, we show regions in the $M_{X_{1}}-\Lambda_{X_{1}}$ plane that can accommodate the observed baryon asymmetry for various choices of $\Lambda_{X_{2}} / \Lambda_{X_{1}}=\Lambda_{c} / \Lambda_{X_{1}}$. In each case, the lower boundary of the viable region is effectively determined by the requirement that $X_{2}$ freezes out with sufficient abundance. As we move upward from this lower boundary, increasing all three $\Lambda$ 's while keeping their ratios fixed, at some point we enter a regime where $X_{2}$ decouples from the SM bath while relativistic, and $Y_{X_{2}}^{\text {fo }}$ saturates at $Y_{X_{2}}^{\mathrm{eq}}\left(T \gg M_{X_{2}}\right)=\left(1 / \pi^{2}\right)\left(T^{3} / s\right)$, so that further increasing the $\Lambda$ 's only reduces $\epsilon_{C P}$ and hence the final $Y_{B}$. Furthermore, for sufficiently high $\Lambda_{X_{2}}$ and $\Lambda_{c}, X_{2}$ dominates the energy density of the Universe before it decays (this does not happen for $X_{1}$ in the parameter space we
Early decay scenario

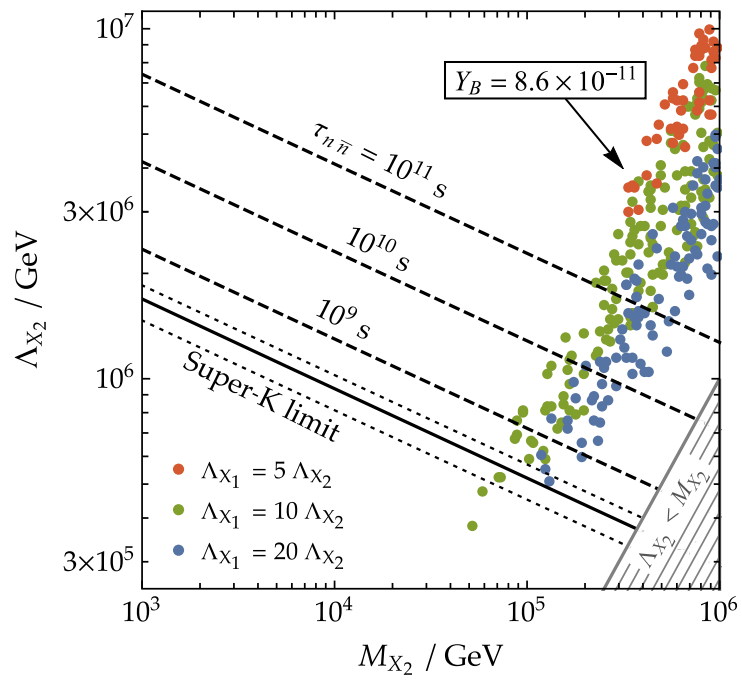

FIG. 3. Parameter space of the minimal EFT probed by $n-\bar{n}$ oscillation for the early decay scenario, assuming that $M_{X_{2}}=4 M_{X_{1}}$. The thicker (solid and dashed) black lines are obtained using central values of nuclear matrix elements from Ref. [61], while the thinner dotted lines show the effect of current lattice calculation uncertainty on the Super-K limit. Points represent solutions with $Y_{B}=8.6 \times 10^{-11}$ found for several fixed $\Lambda_{X_{1}} / \Lambda_{X_{2}}$ ratios while scanning over $\Lambda_{c} \in\left(M_{X_{2}}, \Lambda_{X_{2}}\right)$. The gray shaded region marks $\Lambda_{X_{2}}<M_{X_{2}}$, where EFT validity requires greater than $O(1)$ coupling.

consider), so that its decay injects significant entropy into the plasma, diluting the baryon asymmetry. Both of these effects-saturation and dilution-determine the upper boundary of the viable region.

Early decay scenario.-For the opposite hierarchy $\Lambda_{X_{1}} \gg \Lambda_{X_{2}}, n-\bar{n}$ oscillation is dominated by $X_{2}$ exchange and probes the $M_{X_{2}}-\Lambda_{X_{2}}$ parameter space (see Fig. 3). In this case, $X_{2}$ is short-lived, and its abundance closely follows the equilibrium curve. However, small departures from equilibrium, always present in an expanding Universe because interaction rates are finite, can be sufficient for baryogenesis if washout can be suppressed. The rates for washout processes involving $X_{1}$ and $X_{2}$ are proportional to $n_{1} \Lambda_{X_{1}}^{-4}$ and $n_{2} \Lambda_{X_{2}}^{-4}$, respectively, where $n_{1,2}$ are the number densities of $X_{1,2}$. If $\Lambda_{X_{1}} \sim \Lambda_{X_{2}}$, washout would be efficient until $T \sim M_{X_{1}}$, i.e., until $n_{1}$ starts to fall exponentially. By contrast, by increasing $\Lambda_{X_{1}}$, we enter a regime where washout is dominated by $X_{2}$ processes at high temperatures and becomes inefficient as soon as the temperature falls below $M_{X_{2}}$ (washout due to $u d d \leftrightarrow \bar{u} \bar{d} \bar{d}$, whose rate $\sim T^{11} / M^{2} \Lambda^{8}$ falls steeply with $T$, is also irrelevant at this point), resulting in a short period of baryon asymmetry generation from $X_{2}$ decays (see Fig. 1). Note that increasing $\Lambda_{X_{1}}$ with respect to $\Lambda_{X_{2}}$ also helps to increase departures from equilibrium compared to the degenerate case. 
Figure 3 shows points in the $M_{X_{2}}-\Lambda_{X_{2}}$ plane that can realize the observed $Y_{B}$ through this early decay process for several fixed values of $\Lambda_{X_{1}} / \Lambda_{X_{2}}-5,10$, and 20based on a numerical scan over $M_{X_{2}}<\Lambda_{c}<\Lambda_{X_{2}}$. We find that 5 and 20 are about the smallest and largest $\Lambda_{X_{1}} / \Lambda_{X_{2}}$ ratios that can accommodate the observed $Y_{B}$, while for the majority of the points shown, $\Lambda_{c} \lesssim 3 M_{X_{2}}$. The results can be understood from the competing effects of baryon asymmetry generation and washout, $\Gamma_{\Delta B \neq 0} / \Gamma_{\text {wo }} \sim M^{2} n_{2}\left(\Lambda_{X_{1}}^{2} \Lambda_{X_{2}}^{2} \Lambda_{c}^{2}\right)^{-1} /\left(n_{1} \Lambda_{X_{1}}^{-4}+n_{2} \Lambda_{X_{2}}^{-4}\right) \sim$ $\left(M^{2} / \Lambda_{c}^{2}\right) \times \min \left\{\Lambda_{X_{2}}^{2} / \Lambda_{X_{1}}^{2}, \Lambda_{X_{1}}^{2} / \Lambda_{X_{2}}^{2} e^{-\left(M_{X_{2}}-M_{X_{1}}\right) / T}\right\}$, where the rate of baryon asymmetry generation $\Gamma_{\Delta B \neq 0}$ is calculated from $C P$-violating $X_{2}$ decays. First of all, a lower ratio $\Lambda_{c} / M_{X_{2}}$ is always preferable (within the range of EFT validity), while the ratio $\Lambda_{X_{1}} / \Lambda_{X_{2}}$ has an optimal value of $\sim 10$ as a result of balancing between faster baryon asymmetry generation at higher temperatures (which favors lower $\Lambda_{X_{1}} / \Lambda_{X_{2}}$ ) and later transition to $X_{1}$-dominated washout (which favors a higher $\Lambda_{X_{1}} / \Lambda_{X_{2}}$ ). The requirement of sufficient departure from equilibrium precludes an arbitrarily low $\Lambda_{c}$ and leads to a minimum $M_{X_{2}}$ for this scenario to work, which we see from Fig. 3 is a few $\times 10^{4} \mathrm{GeV}$. Finally, the overall size of $\Lambda_{X_{1,2}}$ is essentially determined by the requirement that $Y_{B}$ freezes out around the time that $\Gamma_{X_{2}}^{\Delta B \neq 0} / \Gamma_{\text {wo }}$ reaches its maximum, and it is higher for a higher $M_{X_{2}}$.

Complementary probes.-In the region of parameter space that is allowed by existing $n-\bar{n}$ oscillation searches, within reach of future experiments such as the ESS and DUNE, and realizes the observed baryon asymmetry, we find that $M_{X_{1,2}} \gtrsim 10^{3}\left(10^{4}\right) \mathrm{GeV}$ in the late (early) decay scenario. Given that $X_{1,2}$ are SM singlets that couple to the SM only via higher dimensional operators, it is unlikely that they can be detected at the LHC or its envisioned upgrades. Likewise, there are no strong flavor physics constraints on our minimal EFT with just the operators in Eq. (3). We note, however, that this outlook can change in a more complicated model that preserves the general features of baryogenesis of our minimal EFT if at least one of $X_{1,2}$ carries SM charges or couples to other fermion species. For example, colored particles at the tera-electron-volt scale, such as the gluino in RPV SUSY, could be within LHC reach. Likewise, extending the exotic fermion couplings to other quark flavors can introduce potential constraints from flavor violation considerations such as $K^{0}-\bar{K}^{0}$ mixing [70]. Nevertheless, our minimal EFT study illustrates that $n-\bar{n}$ oscillation might be uniquely placed to probe realistic baryogenesis scenarios that are inaccessible via other searches.

Conclusions. - Establishing baryon number violation (or the absence thereof up to a certain scale) will have farreaching implications on our understanding of fundamental particle interactions, in particular on the mechanism that generates the observed baryon asymmetry in our Universe. Motivated by the unprecedented sensitivity to $n-\bar{n}$ oscillation that can be achieved at future facilities, the ESS and DUNE in particular, which offers new opportunities to probe $|\Delta B|=2, \Delta L=0$ interactions, we studied implications of a potential discovery for baryogenesis scenarios involving $n-\bar{n}$ mediators. We took a bottom-up EFT approach with a minimal set of four-fermion operators coupling the $n-\bar{n}$ mediators to the SM, which, despite being simplistic, sets a useful template that more sophisticated theories can build upon. We identified two viable baryogenesis scenarios-one involving late out-of-equilibrium decays of a heavy Majorana fermion, and another involving earlier decays assisted by a suppressed washout rate- that can be realized in the parameter space to be probed by future $n-\bar{n}$ oscillation searches, with no corresponding collider or flavor signals. These results highlight the capability of $n-\bar{n}$ oscillation experiments to probe an important BSM phenomenon, that of baryogenesis, beyond the scope of other searches.

We thank J. Barrow, G. Brooijmans, C. Csáki, and E. Rinaldi for the helpful comments and discussions. C. G. was supported by the European Commission through Marie Curie Career Integration Grant No. 631962, and by the Helmholtz Association. B. S. was partially supported by NSF CAREER Grant No. PHY-1654502 and thanks the CERN and DESY theory groups, where part of this work was conducted, for the hospitality. The work of J. D. W. was supported by DOE Grant No. DE-SC0007859 and the Humboldt Research Award. The work of Z.Z. was supported by DOE Grant No. DE-SC0007859, the Rackham Dissertation Fellowship, and the Summer Leinweber Research Award. J. D. W. and Z. Z. also thank the DESY theory group for the hospitality. This work was performed in part at the Aspen Center for Physics, which was supported by National Science Foundation Grant No. PHY-1066293.

[1] K. Abe et al. (Super-Kamiokande Collaboration), Search for proton decay via $p \rightarrow e^{+} \pi^{0}$ and $p \rightarrow \mu^{+} \pi^{0}$ in 0.31 megaton years exposure of the Super-Kamiokande water Cherenkov detector, Phys. Rev. D 95, 012004 (2017).

[2] K. Abe et al. (Super-Kamiokande Collaboration), Search for nucleon decay into charged antilepton plus meson in 0.316 megaton years exposure of the Super-Kamiokande water Cherenkov detector, Phys. Rev. D 96, 012003 (2017).

[3] A. Kobach, Baryon number, lepton number, and operator dimension in the standard model, Phys. Lett. B 758, 455 (2016).

[4] D. G. Phillips II et al., Neutron-antineutron oscillations: Theoretical status and experimental prospects, Phys. Rep. 612, 1 (2016).

[5] Other $|\Delta B|=2, \Delta L=0$ processes include dinucleon decays: $n n \rightarrow \pi^{0} \pi^{0}, p p \rightarrow \pi^{+} \pi^{+}, p n \rightarrow \pi^{+} \pi^{0}$ probe the same operators as $n-\bar{n}$ oscillation, but with a lower sensitivity at 
present [J. Gustafson et al. (Super-Kamiokande Collaboration), Search for dinucleon decay into pions at SuperKamiokande, Phys. Rev. D 91, 072009 (2015)]; while $p p \rightarrow K^{+} K^{+}[$M. Litos et al., Search for Dinucleon Decay into Kaons in Super-Kamiokande, Phys. Rev. Lett. 112, 131803 (2014)]; can be relevant for $B$-violating new physics with suppressed couplings to first-generation quarks [C. Csaki, Y. Grossman, and B. Heidenreich, MFV SUSY: A natural theory for $R$-parity violation, Phys. Rev. D 85, 095009 (2012); K. Aitken, D. McKeen, T. Neder, and A. E. Nelson, Baryogenesis from oscillations of charmed or beautiful baryons, Phys. Rev. D 96, 075009 (2017)].

[6] M. Baldo-Ceolin et al., A new experimental limit on neutron-antineutron oscillations, Z. Phys. C 63, 409 (1994).

[7] K. Abe et al. (Super-Kamiokande Collaboration), The search for $n-\bar{n}$ oscillation in Super-Kamiokande I, Phys. Rev. D 91, 072006 (2015).

[8] D. Milstead, A new high sensitivity search for neutronantineutron oscillations at the ESS, Proc. Sci., EPSHEP2015 (2015) 603 [arXiv:1510.01569].

[9] M. J. Frost (NNbar Collaboration), The NNbar experiment at the European Spallation Source, arXiv:1607.07271.

[10] A. R. Young, Prospects and progress: New experimental searches for neutron-antineutron oscillations and related probes for new physics, https://indico.ill.fr/indico/event/87/ session/8/contribution/101.

[11] J. E. T. Hewes, Searches for bound neutron-antineutron oscillation in liquid argon time projection chambers, Report No. FERMILAB-THESIS-2017-27 (2017).

[12] J. Barrow, Neutron-antineutron transformation at the deep underground neutrino experiment 2018, https://indico.ill.fr/ indico/event/87/session/8/contribution/12.

[13] V. A. Kuzmin, V. A. Rubakov, and M. E. Shaposhnikov, On the anomalous electroweak Baryon number nonconservation in the early Universe, Phys. Lett. 155B, 36 (1985).

[14] I. Affleck and M. Dine, A new mechanism for baryogenesis, Nucl. Phys. B249, 361 (1985).

[15] M. Fukugita and T. Yanagida, Baryogenesis without grand unification, Phys. Lett. B 174, 45 (1986).

[16] A. G. Cohen, D. B. Kaplan, and A. E. Nelson, Progress in electroweak baryogenesis, Annu. Rev. Nucl. Part. Sci. 43, 27 (1993).

[17] A. Riotto, Theories of baryogenesis, arXiv:hep-ph/9807454.

[18] A. Riotto and M. Trodden, Recent progress in baryogenesis, Annu. Rev. Nucl. Part. Sci. 49, 35 (1999).

[19] W. Bernreuther, $C P$ violation and baryogenesis, Lect. Notes Phys. 591, 237 (2002).

[20] M. Dine and A. Kusenko, Origin of the matter-antimatter asymmetry, Rev. Mod. Phys. 76, 1 (2003).

[21] W. Buchmuller, P. Di Bari, and M. Plumacher, Leptogenesis for pedestrians, Ann. Phys. (Amsterdam) 315, 305 (2005).

[22] J. M. Cline, Baryogenesis, arXiv:hep-ph/0609145.

[23] S. Davidson, E. Nardi, and Y. Nir, Leptogenesis, Phys. Rep. 466, 105 (2008).

[24] D. E. Morrissey and M. J. Ramsey-Musolf, Electroweak baryogenesis, New J. Phys. 14, 125003 (2012).

[25] K. S. Babu, R. N. Mohapatra, and S. Nasri, Postsphaleron Baryogenesis, Phys. Rev. Lett. 97, 131301 (2006).
[26] K. S. Babu, R. N. Mohapatra, and S. Nasri, Unified TeV Scale Picture of Baryogenesis and Dark Matter, Phys. Rev. Lett. 98, 161301 (2007).

[27] K. S. Babu, P. S. Bhupal Dev, and R. N. Mohapatra, Neutrino mass hierarchy, neutron-anti-neutron oscillation from baryogenesis, Phys. Rev. D 79, 015017 (2009).

[28] R. Allahverdi, B. Dutta, and K. Sinha, Baryogenesis and late-decaying moduli, Phys. Rev. D 82, 035004 (2010).

[29] P.H. Gu and U. Sarkar, Baryogenesis and neutronantineutron oscillation at TeV, Phys. Lett. B 705, 170 (2011).

[30] P. H. Gu and U. Sarkar, Inflationary baryogenesis with low reheating temperature and testable neutron-antineutron oscillation, arXiv:1110.2926.

[31] K. S. Babu and R. N. Mohapatra, Coupling unification, GUT-scale baryogenesis and neutron-antineutron oscillation in SO(10), Phys. Lett. B 715, 328 (2012).

[32] N. Bernal, F. X. Josse-Michaux, and L. Ubaldi, Phenomenology of WIMPy baryogenesis models, J. Cosmol. Astropart. Phys. 01 (2013) 034.

[33] J. M. Arnold, B. Fornal, and M. B. Wise, Simplified models with baryon number violation but no proton decay, Phys. Rev. D 87, 075004 (2013).

[34] K. S. Babu, P. S. Bhupal Dev, E. C. F. S. Fortes, and R. N. Mohapatra, Post-sphaleron baryogenesis and an upper limit on the neutron-antineutron oscillation time, Phys. Rev. D 87, 115019 (2013).

[35] R. Allahverdi and B. Dutta, Natural GeV dark matter and the baryon-dark matter coincidence puzzle, Phys. Rev. D 88, 023525 (2013).

[36] S. Patra and P. Pritimita, Post-sphaleron baryogenesis and $n-\bar{n}$ oscillation in non-SUSY $\mathrm{SO}(10)$ GUT with gauge coupling unification and proton decay, Eur. Phys. J. C 74, 3078 (2014).

[37] E. Herrmann, On baryogenesis and $n \bar{n}$-oscillations, arXiv: 1408.4455

[38] P. S. B. Dev and R. N. Mohapatra, TeV scale model for baryon and lepton number violation and resonant baryogenesis, Phys. Rev. D 92, 016007 (2015).

[39] A. Ghalsasi, D. McKeen, and A. E. Nelson, Baryogenesis via mesino oscillations, Phys. Rev. D 92, 076014 (2015).

[40] P. H. Gu, E. Ma, and U. Sarkar, Connecting radiative neutrino mass, neutron-antineutron oscillation, proton decay, and leptogenesis through dark matter, Phys. Rev. D 94, 111701 (2016).

[41] P. H. Gu and U. Sarkar, High-scale baryogenesis with testable neutron-antineutron oscillation and dark matter, Phys. Rev. D 96, 031703 (2017).

[42] L. Calibbi, E. J. Chun, and C.S. Shin, LSP baryogenesis and neutron-antineutron oscillations from $R$-parity violation, J. High Energy Phys. 10 (2017) 177.

[43] R. Allahverdi, P. S. B. Dev, and B. Dutta, A simple testable model of baryon number violation: Baryogenesis, dark matter, neutron-antineutron oscillation and collider signals, Phys. Lett. B 779, 262 (2018).

[44] G. Fidecaro et al. (CERN-GRENOBLE-PADUA-RUTHERFORD-SUSSEX Collaboration), Experimental search for neutron anti-neutron transitions with free neutrons, Phys. Lett. 156B, 122 (1985). 
[45] G. Bressi et al., Final results of a search for free neutron anti-neutron oscillations, Nuovo Cimento Soc. Ital. Fis. A 103, 731 (1990).

[46] T. W. Jones et al. (Irvine-Michigan-Brookhaven Collaboration), Search for $N \bar{N}$ Oscillation in Oxygen, Phys. Rev. Lett. 52, 720 (1984).

[47] M. Takita et al. (Kamiokande Collaboration), Search for neutron-anti-neutron oscillation in a ${ }^{16} \mathrm{O}$ nucleus, Phys. Rev. D 34, 902(R) (1986).

[48] C. Berger et al. (Frejus Collaboration), Search for neutronanti-neutron oscillations in the Frejus detector, Phys. Lett. B 240, 237 (1990).

[49] J. Chung et al., Search for neutron anti-neutron oscillations using multiprong events in Soudan 2, Phys. Rev. D 66, 032004 (2002).

[50] B. Aharmim et al. (SNO Collaboration), Search for neutronantineutron oscillations at the Sudbury Neutrino Observatory, Phys. Rev. D 96, 092005 (2017).

[51] L. N. Chang and N.P. Chang, $B-L$ Nonconservation and neutron oscillation, Phys. Lett. 92B, 103 (1980).

[52] T. K. Kuo and S. T. Love, Neutron Oscillations and the Existence of Massive Neutral Leptons, Phys. Rev. Lett. 45, 93 (1980).

[53] S. Rao and R. Shrock, $n \leftrightarrow \bar{n}$ transition operators and their matrix elements in the MIT bag model, Phys. Lett. 116B, 238 (1982).

[54] S. Rao and R. E. Shrock, Six fermion $(B-L)$ violating operators of arbitrary generational structure, Nucl. Phys. B232, 143 (1984).

[55] W. E. Caswell, J. Milutinovic, and G. Senjanovic, Matterantimatter transition operators: A manual for modeling, Phys. Lett. 122B, 373 (1983).

[56] M. I. Buchoff and M. Wagman, Perturbative renormalization of neutron-antineutron operators, Phys. Rev. D 93, 016005 (2016).

[57] See Supplemental Material at http://link.aps.org/ supplemental/10.1103/PhysRevLett.121.171801 for a brief review of $n-\bar{n}$ oscillation operators and details of the baryogenesis calculation.

[58] K. G. Chetyrkin, J. H. Kuhn, and M. Steinhauser, RunDEC: A Mathematica package for running and decoupling of the strong coupling and quark masses, Comput. Phys. Commun. 133, 43 (2000).
[59] M. I. Buchoff, C. Schroeder, and J. Wasem, Neutronantineutron oscillations on the lattice, Proc. Sci., LATTICE2012 (2012) 128 [arXiv:1207.3832].

[60] S. Syritsyn, M. I. Buchoff, C. Schroeder, and J. Wasem, Neutron-antineutron oscillation matrix elements with domain wall fermions at the physical point, Proc. Sci., LATTICE2015 (2015) 132.

[61] E. Rinaldi, S. Syritsyn, M. L. Wagman, M. I. Buchoff, C. Schroeder, and J. Wasem, Neutron-antineutron oscillations from lattice QCD, arXiv:1809.00246.

[62] D. V. Nanopoulos and S. Weinberg, Mechanisms for cosmological baryon production, Phys. Rev. D 20, 2484 (1979).

[63] F. Rompineve, Weak scale baryogenesis in a supersymmetric scenario with $R$-parity violation, J. High Energy Phys. 08 (2014) 014.

[64] Y. Cui, Natural baryogenesis from unnatural supersymmetry, J. High Energy Phys. 12 (2013) 067.

[65] G. Arcadi, L. Covi, and M. Nardecchia, Gravitino dark matter and low-scale baryogenesis, Phys. Rev. D 92, 115006 (2015).

[66] Our minimal EFT bears similarities with the models studied in [70] and [I. Baldes, N. F. Bell, A. Millar, K. Petraki, and R. R. Volkas, The role of $C P$ violating scatterings in baryogenesis: Case study of the neutron portal, J. Cosmol. Astropart. Phys. 11 (2014) 041]. However, these papers focused on baryogenesis using operators of the form $\left(\bar{d}^{c} P_{R} d\right)\left(\bar{u}^{c} P_{R} X\right)$, which, upon Fierz transformations, are equivalent to generation-antisymmetric components of the $\left(\bar{u}^{c} P_{R} d\right)\left(\bar{d}^{c} P_{R} X\right)$ operators in Eq. (3) and thus do not mediate $n-\bar{n}$ oscillation at tree level.

[67] A. D. Sakharov, Violation of $C P$ invariance, $C$ asymmetry, and baryon asymmetry of the Universe, Pis'ma $\mathrm{Zh}$. Eksp. Teor. Fiz. 5, 32 (1967);[JETP Lett. 5, 24 (1967)]; Usp. Fiz. Nauk 161, 61 (1991); [Sov. Phys. Usp. 34, 392 (1991)].

[68] P. A. R. Ade et al. (Planck Collaboration), Planck 2013 results. XVI. Cosmological parameters, Astron. Astrophys. 571, A16 (2014).

[69] P. A. R. Ade et al. (Planck Collaboration), Planck 2015 results. XIII. Cosmological parameters, Astron. Astrophys. 594, A13 (2016).

[70] C. Cheung and K. Ishiwata, Baryogenesis with higher dimension operators, Phys. Rev. D 88, 017901 (2013). 